\title{
Head to head comparison of the clinical and cost effectiveness of Observe and Plan versus PRN for the treatment of wet macular degeneration
}

\author{
James Wawrzynski ${ }^{*}$, Ramiro Salom², Anna Pouncey ${ }^{3}$, Marilyn Martin ${ }^{2}$ and Mehmet Ozturk ${ }^{2}$ \\ ${ }^{1}$ Moorfields Eye Hospital: 162 City Road, London, UK \\ ${ }^{2}$ Barking, Havering and Redbridge University Hospitals: Rom Valley Way, Romford, Essex. UK \\ ${ }^{3}$ Royal Free Hospital: Pond St, Hampstead, London, UK
}

\begin{abstract}
Purpose: "Observe and Plan" (O\&P) is a novel treatment regimen for neovascular AMD (nAMD). It aims to optimise the intravitreal anti-VEGF injection frequency whilst dramatically reducing the number of clinical reviews (compared to PRN/ treat and extend). However, there has been no head-to-head comparison of O\&P versus the other regimens: We aim to establish whether O\&P is non-inferior to PRN and whether there is an economic advantage.

Methods: Prospective interventional cohort study: Patients undergoing treatment with PRN aflibercept for nAMD were enrolled. All were switched to O\&P aflibercept for one year. Best corrected visual acuity (BCVA) and central macular thickness (CMT) were recorded in addition to the number of clinic visits and intravitreal injections. Their results were compared in a paired analysis to their own data whilst on PRN treatment the preceding year.
\end{abstract}

Results: 25 patients were enrolled. 17 completed one year of treatment, one of whom was subsequently excluded (confounding pathology). Analysis of area under the curve for BCVA and CMT found O\&P to be non-inferior to PRN $\mathrm{p}=0.03, \mathrm{p}<0.01$.

The mean number of clinical reviews was significantly reduced under O\&P (1.80 (SD 0.68) vs. 8.66 (SD 0.72) visits per year; $\mathrm{P}=<0.01)$ with an improvement in injection to visit ratio (1.01 vs. 1.67 vists/injection), equating to an estimated saving of $£ 1,228$ per patient per year.

Conclusion: The O\&P regimen for treatment of nAMD was clinically non-inferior to PRN. The O\&P regimen was superior to PRN in terms of reducing the health economic burden and treatment burden of nAMD.

\section{Introduction}

In 2014, data were published supporting a new regimen, "observe and plan" (O\&P), which aims to optimise the interval between injections individually to each patient whilst also significantly reducing the frequency of clinical reviews [1]. The O\&P regimen was designed based on the observation by Mantel et al in 2013 that patients with neovascular age related macular degeneration (nAMD) tend to exhibit a highly predictable and regular need for re-treatment [2]. In the O\&P regimen, injections are planned ahead in groups of several at a time; patients are reviewed clinically after every set of injections and a decision is then taken to increase, decrease or maintain the injection frequency as appropriate for the next series of injections. As in the "treat and extend" (T\&E) regimen, the interval is extended up to a maximum of 12 weeks if the macula is free of intraretinal or subretinal fluid, however if there is a recurrence of oedema then the interval is shortened to a minimum of 4 weeks $[3,4]$.

Currently there is evidence from two non-controlled interventional case series that O\&P is a safe and effective regimen for ranibizumab and aflibercept injections in the first two years from the time of diagnosis $[3,4]$. Given the promising recent results of studies investigating O\&P when initiated for newly diagnosed patients, the question of switching the vast numbers of patients currently in AMD clinics on pro re nata $(\mathrm{PRN}) / \mathrm{T} \& \mathrm{E} /$ fixed interval regimens to O\&P arises. There is however no comparative evidence for the non-inferiority of $\mathrm{O} \& \mathrm{P}$ when nAMD patients are switched from another regimen after several years of treatment. In this work, we aim to establish whether O\&P is non-inferior to PRN when patients undergoing treatment with PRN aflibercept are switched to O\&P.

\section{Methods}

\section{Study design}

This prospective interventional cohort study was conducted by the medical retina department at our centre. All patients provided informed consent prior to enrolment. Functional and anatomical outcomes in patients undergoing treatment on the $\mathrm{O} \& \mathrm{P}$ regimen were recorded over approximately 12 months, as well as the number of clinical visits and injections required. In order to assess for non-inferiority and cost-effectiveness of the O\&P treatment regimen, a retrospective case-control comparison was then performed; each patient's data were

${ }^{\star}$ Correspondence to: James Wawrzynski, Moorfields Eye Hospital: 162 City Road, London, EC1V 2PD, UK, E-mail: james.wawrzynski@cantab.net

Key words: macular degeneration, CNVM, anti-VEGF, observe and plan

Received: May 13, 2020; Accepted: June 01, 2020; Published: June 05, 2020 
matched against their results recorded in preceding 12-month period, whilst on PRN treatment.

\section{Inclusion \& exclusion criteria}

Patients greater than or equal of 50 years of age, with an established diagnosis of nAMD on fundus fluorescein angiogram, who had received PRN anti-VEGF injections of aflibercept (Eylea) for nAMD for 18 months or more, were eligible for inclusion. Patients were required to have a baseline best corrected visual acuity higher than logMAR 1.3 and have had no change to their regimen within the last 6 months.

Patients unable or unwilling to provide informed consent were excluded. Patients with co-existing macular pathology or co-existing best corrected visual acuity (BCVA)-affecting ocular pathology were excluded. Those with cataract were excluded only if severe enough to prevent sufficient quality of retinal images and OCT scanning of the macula. Patients with choroidal neovascularisiation not resulting from nAMD, or a history of uveitis in the study eye, prior vitrectomy or aphakia, were also excluded.

Patients were withdrawn from the study if an unexpected progression of $\mathrm{nAMD}$ required a deviation from the injection protocol in the "observe and plan" regimen. Such examples may include a clinical decision to cease anti-VEGF treatment due to futility or the need for more frequent injections than specified by the protocol. Patients could also be withdrawn following development of co-existing macular or other vision limiting pathology, if there was a 2 line change in their visual acuity following cataract extraction or following removal of patient consent.

\section{Observe and plan regimen}

Patients enrolling in the trial received a new integrated care pathway added to their notes specifically for "observe and plan" and were treated with O\&P aflibercept (Eylea). The O\&P protocol has previously been described in the literature [1] and is summarised in Figure 1 and below.

In order to determine the treatment interval, the number of weeks between the patients' most recent two injections on the PRN regimen was calculated. If there was no oedema prior to the last injection, this became the patient's injection interval for the next four injections. If there was oedema prior to the last injection, the shortest interval between injections in the last six months that resulted in exudation was calculated and two weeks subtracted to determine the patient's injection interval. Injection interval could be set at a minimum of 4 and maximum of 12 weeks. Patients received a clinical review at the time of the fourth injection. If the macula was dry, the injection interval was extended by 2 weeks. In the presence of macular oedema, the injection interval was reduced by two weeks. This was repeated cyclically for a total of one year.

\section{Clinical investigations}

The enrolment visit and all clinical reviews included a patient consult, best corrected visual acuity (BCVA) using the Early Treatment of Diabetic Study (ETDRS) chart, IOP (iCare TA01i model), slit lamp examination, and spectral domain optical coherence tomography (OCT) scan of the macula (Topcon - Tokyo, Japan). Each injection visit consisted of an intravitreal injection of aflibercept, in accordance with RCOphth guidelines. BCVA and an OCT scan of the macula were also recorded.

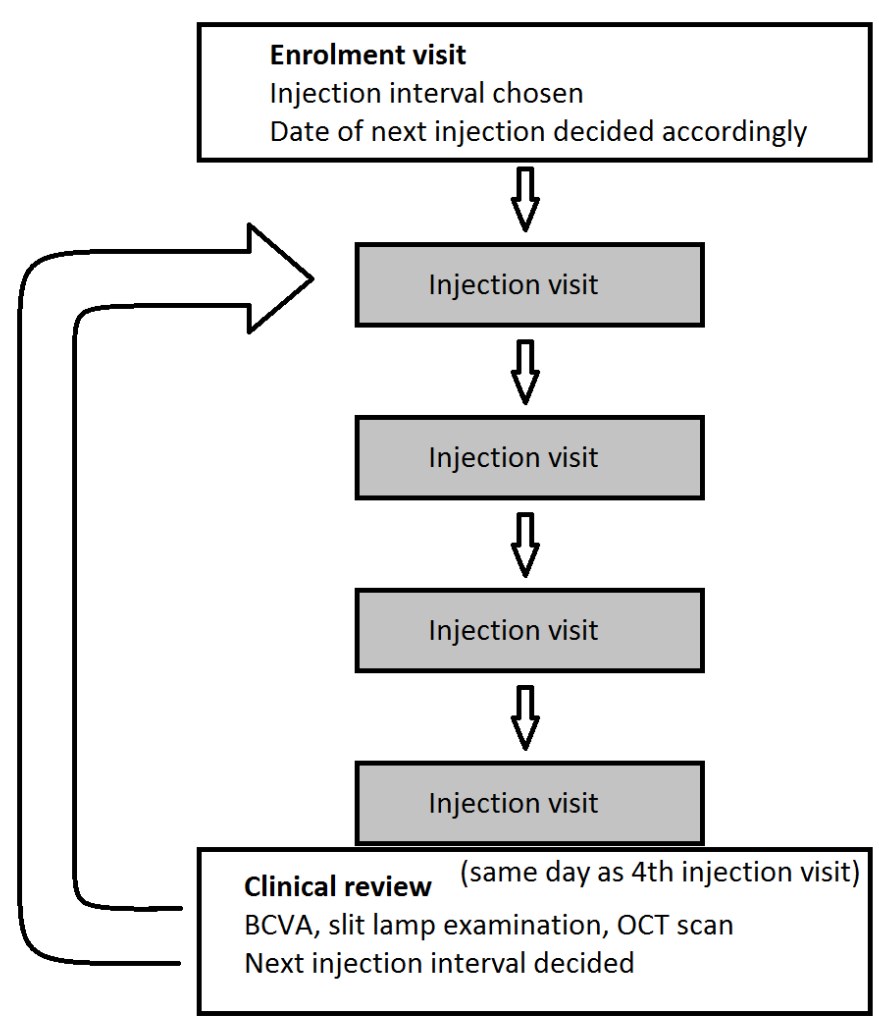

Figure 1. Observe and Plan injection and review protocol

\section{Patient safety}

To reduce any risk to vision, all patients received a BCVA/ OCT review at each injection visit and clinical review. These would not ordinarily be required as part of the O\&P regimen and only results obtained during a "clinic visit" were used to inform the treatment interval. A change in BCVA of 2 lines of greater, an increase in macular oedema on OCT, or any nursing or patient concerns prompted review by an ophthalmologist, who could instigate a change to the injection interval if necessary. If the injection interval was changed the patient was withdrawn from the trial at that stage.

\section{Statistical analysis}

Our aim was to establish whether O\&P is non-inferior to PRN when patients are switched from PRN to O\&P by monitoring the visual acuity, central macular thickness, presence of macular intraretinal/ subretinal fluid and subretinal fibrosis. In addition we performed an economic analysis by studying the patients' injection interval and number of clinical reviews required. O\&P was the intervention group and a control group were established by performing a retrospective paired analysis, comparing each patient's outcomes to their own results whilst they were on the PRN regimen over the preceding year.

Data was collated from each monitoring and injection visit. The primary outcomes assessed were BCVA and central retinal thickness within the central $1 \mathrm{~mm}$ subfield over 12 months. For the purpose of the non-inferiority test, clinically significant worsening of vision was set at one line and clinically significant worsening of macular oedema was set at 50 micrometers. The secondary outcomes were as follows: Clinical; proportion of eyes suffering a visual acuity loss of 15 letters or greater, proportion of eyes with macular oedema at injection visits, proportion 
of eyes that had developed subretinal fibrosis. Economic; total number of injections, average injection interval, number of clinical reviews.

Statistical analysis was conducted using $\mathrm{R}$ studio. Parametric continuous data was expressed as the mean (+/- standard deviation), non-parametric continuous as median (range) and categorical data as number (percentage).

Analysis of change over time in BCVA and central macular thickness (CMT) were conducted by analysing the area under the curve standardised to 1 year. Area under the curve was calculated using the trapezoidal rule for each change in BCVA and CMT from baseline. The baseline measurements for O\&P were determined at the enrolment visit. The baseline measurements for PRN were considered to be those taken at the patient's clinic visit one year prior to enrolment. A Shapiro-Wilk test for normality was performed on the resulting data in order to determine the appropriateness of parametric/ nonparametric hypothesis testing. Non-inferiority testing was conducted with paired t-tests and paired Wilcoxon signed rank tests. Comparison of proportional data, (i.e. visual loss above threshold), was conducted with Fisher's exact test. Cost analysis, standardised to 1 year, was conducted utilising local trust costing for each clinical encounter, and pharmaceutical cost of the anti-VEGF agents.

\section{Ethical approval}

The study conformed to the Helsinki Declaration of 1975 (revised 1983) and underwent institutional board review and approval.

\section{Results}

A total of 25 patients were enrolled. All patients were being treated with PRN aflibercept at the time of enrolment and were switched onto O\&P aflibercept. 17 patients (72\%) completed one year of treatment on the O\&P regimen with aflibercept (Eylea); one of whom was subsequently excluded from further analysis due to cataract surgery confounding visual acuity measurement. The remaining 16 patients were included in the analysis. A matched historical control group was formed using results from the same 16 patients whilst on the PRN regimen the preceding year.

Eight patients did not complete one year on the O\&P regimen and were withdrawn from the study. Reasons for withdrawal were as follows: Three patients were unable to attend their scheduled appointments resulting in a deviation from the protocol. One patient withdrew consent. One patient underwent a change in their antiVEGF treatment as a result of an ischaemic stroke and was therefore withdrawn. One patient passed away as a result of an unrelated malignancy. One patient was treated more frequently than permitted by the protocol as a result of macular haemorrhage and increasing metamorphopsia despite no increase in macular oedema. One patient developed a cataract that precluded OCT scanning of the retina.

\section{Patient demographics and baseline characteristics}

The mean age of patients at enrolment was 83.0 years old (SD 6.3 , range $73-94) .69 \%$ of patients were female. Mean visual acuity at baseline was 0.51 (logMAR), (SD 0.23, range $0.04-0.92) .88 \%$ of patients were pseudophakic at enrolment. Median time since diagnosis of wet age related macular degeneration was 5 years and 1 month (range:2 years and 1 month -8 years and 5 months). In the control group mean visual acuity at baseline was 0.50 (logMAR) (SD 0.33 , range: $0.10-1.30)$.

\section{Clinical outcomes}

Visual acuity: The mean best corrected visual acuity (BCVA) in the O\&P group fell from 0.51 to $0.59 \log$ MAR during the course of the study. In the preceding year the same patients had a mean BCVA drop from 0.50 to $0.51 \log$ MAR whilst on the PRN regimen (Figure 2).

The area under the curve (AUC) for BCVA in the O\&P group (standardised to 1 year of follow-up) was 0.225 (change in Baseline VA, logMar), with an average change in VA (AUC) per patient of 0.0141 . Under PRN in the preceding year, the same patients had an AUC (standardised to 1 year of follow-up) of 0.035 (change in Baseline VA, logMar), with an average change in VA (AUC) per patient of 0.00218 . A paired t-test to assess for non-inferiority of O\&P compared to PRN was conducted with a clinically significant threshold for loss of visual acuity set at 1 line of vision. O\&P was found to be non-inferior to PRN $(p=0.03)$. An additional Wilcoxon signed rank test for inferiority was performed and confirmed the $t$-test result to be robust $(\mathrm{p}=0.005)$. The average visit-to-visit fluctuation in visual acuity was 0.08 under $\mathrm{O} \& \mathrm{P}$ and 0.09 under PRN. A two-sided t-test did not find any statistically significant difference between the groups.

Overall, $3 / 16(18.8 \%)$ patients lost 15 letters or more in the O\&P group compared to $1 / 16(6.3 \%)$ in the PRN group. Fisher's exact test did not find any statistically significant difference between these values $(\mathrm{p}=0.30)$.

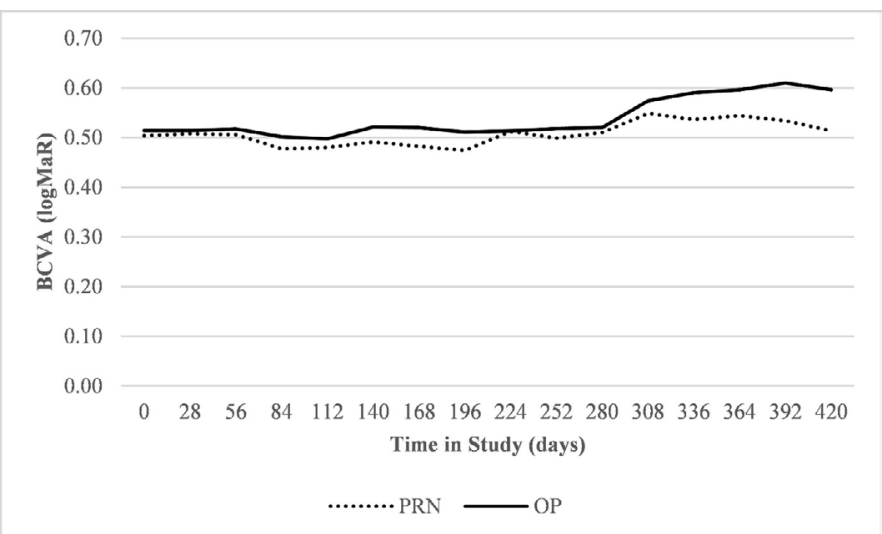

Figure 2. Trend of mean BCVA $(\log M A R)$ shown at 4 week intervals over the course of the study under both the PRN and O\&P regimes

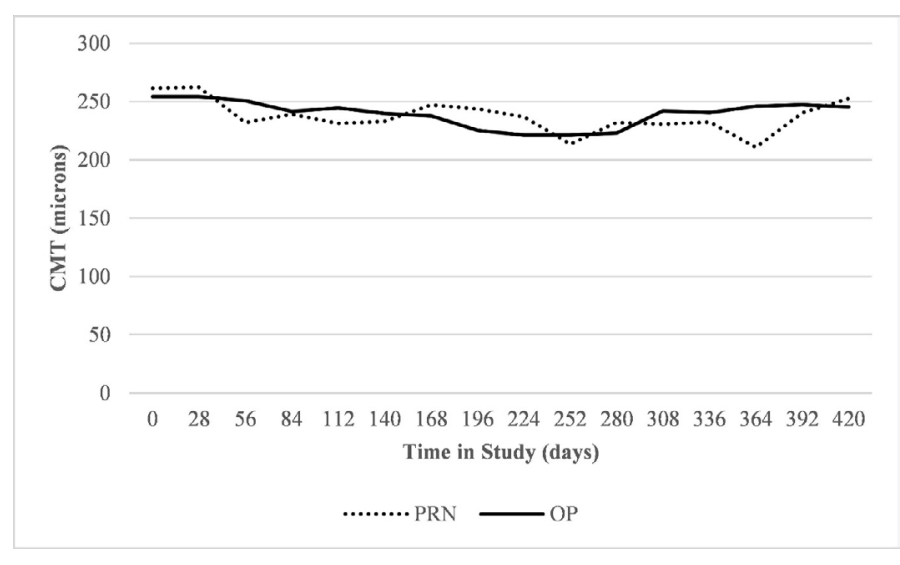

Figure 3. Trend of mean CMT (microns) shown at 4 week intervals over the course of the study under both the PRN and O\&P regimes 
Macular oedema: The mean CMT in the O\&P group was 254 micrometres at enrolment decreasing to 246 micrometres over the course of the study. The mean CMT in the PRN group was 261 micrometres at enrolment decreasing to 254 micrometres over the course of the study (Figure 3).

The AUC of the CMT in the O\&P group (standardised to 1 year of follow-up) was -313 (change from baseline CMT, micrometres), with an average change per patient of -19.56 . Under PRN in the preceding year, the same patients had an AUC (standardised to 1 year of followup) of -424 (change from baseline CMT, micrometres), with an average change per patient of -26.5. A paired t-test to assess for non-inferiority of O\&P compared to PRN was conducted with a clinically significant threshold for worsening of macular oedema set at 50 micrometres. O\&P was found to be non-inferior to PRN ( $\mathrm{p}<0.01)$.

The presence or absence of intraretinal or subretinal fluid was recorded prior to each injection. Oedema was found at $99 \%$ of visits under the PRN regimen and $76 \%$ of visits under O\&P (Figure 4). Fisher's exact test found a statistically significant difference between these values $(\mathrm{p}<0.01)$, with macular oedema significantly less likely to be present in $\mathrm{O} \& \mathrm{P}$ patients.

Subretinal fibrosis: All patients had subretinal fibrosis at enrolment therefore no further analysis was performed on the development of subretinal fibrosis during treatment with the O\&P or PRN regimens.

\section{Economic analysis}

Number of clinical reviews: The mean number of clinical reviews (including an OCT scan, examination by doctor and decision regarding the intravitreal injection treatment interval) was 1.80 (SD 0.68) per year under the O\&P regimen and 8.66 (SD 0.72) per year under PRN. A paired t-test demonstrated that significantly fewer clinical reviews were required under $\mathrm{O} \& \mathrm{P}$ than under $\mathrm{PRN}(\mathrm{p}<0.01)$. Based on a cost of $£ 117$ for an OCT scan and $£ 62$ for a follow-up consultation at our trust, this equates to a saving of $£ 1,228$ per patient per year.

Number of injections: The mean number of injections required per year was 5.6 (SD 0.95) in the O\&P group and 5.19 (SD 1.01) in the PRN group. A paired t-test found no statistically significant difference between these values $(\mathrm{p}=0.52)$. The mean number of visits required per injection under O\&P was 1.01. The mean number of visits required per injections under PRN was 1.67 (figure 5). A paired t-test confirmed that there is a statistically significant difference between these values $(\mathrm{p}<0.01)$.

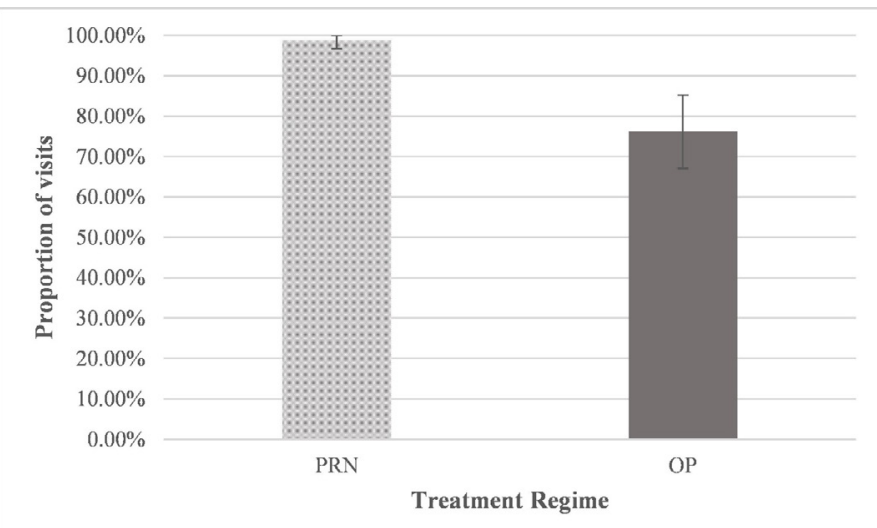

Figure 4. Proportion of patient visits where subretinal/ intraretinal fluid was present under both the PRN and O\&P regimes

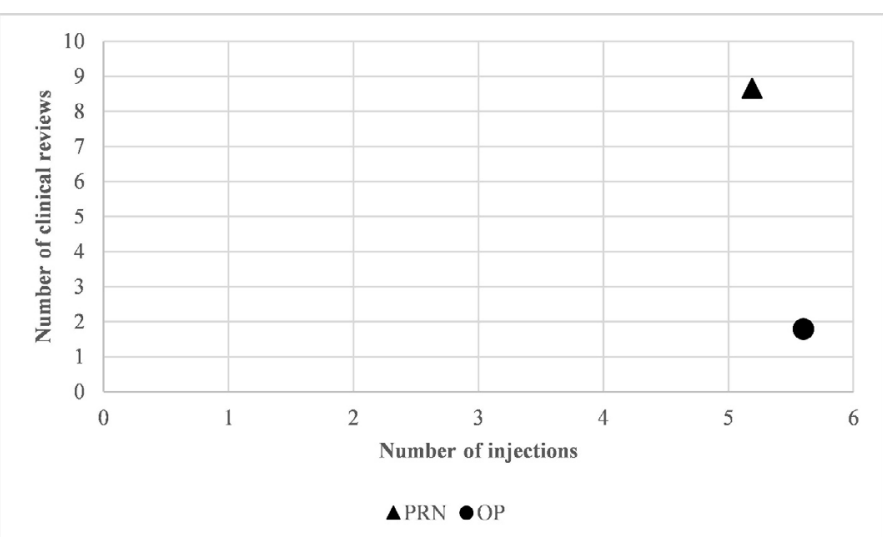

Figure 5. Number of clinical reviews vs number of injections under the PRN and O\&P regimes

\section{Discussion}

The number of patients with neovascular age related macular degeneration undergoing regular treatment with anti-VEGF injections is rising rapidly [5]. The capacity of health care services to service the demand is being stretched [6]. Anti-VEGF injections are well established as the most effective therapy for nAMD [7] however determining the optimal injection frequency has proved to be a challenge; each patient can tolerate a different maximal interval between injections and the optimal interval can change throughout the course of the patient's disease [8]. The key regimens in use are T\&E, PRN and fixed interval dosing [9-11].

Regular injections and clinical reviews (monitoring the patient with a visual acuity test, OCT scan and clinical examination) at each visit represent a growing and unsustainable financial burden for healthcare systems around the world $[5,6]$. In addition, regular visits to the eye clinic are disruptive for patients. Each visit can require the patient to wait several hours for a clinical assessment followed by a possible injection. The number of injections received on the fixed interval regimen is likely to be significantly superfluous to requirements for most patients and has therefore largely been abandoned after one year of treatment in the UK. PRN also requires regular attendance at the eye clinic 4 or 6 weekly (for Ranibizumab and Aflibercept respectively) for clinical review and the Treat and Extend regimen requires a clinical review after every injection (at 4 or 6 weekly intervals for ranibizumab/ aflibercept respectively up to a maximum interval of 12 weekly).

Our study is the first controlled interventional cohort study to investigate the safety and economic benefits of switching longstanding nAMD patients from the PRN regimen to O\&P in a real world setting. It also provides the first head to head comparison of O\&P against any other established treatment regimen. Despite reducing the number of clinical reviews five-fold, patient's average visual acuity, chance of significant vision loss ( $>15$ letter loss) and central macular thickness were equivalent (non-inferior) under the O\&P regimen when compared to PRN. Patients were significantly less likely to have recurrences of macular oedema on O\&P as compared to PRN. The annual number of injections patients received (and therefore the average treatment interval) remained the same under O\&P as it was under PRN.

Previous non-controlled studies have investigated O\&P for treatment naïve patients and have demonstrated good clinical efficacy with patients achieving an initial letter gain of 8.7 letters [3], similar to the results found in ANCHOR/ MARINA, which was maintained for at least two years. Our findings are in agreement with this previous 
evidence and show that most patients were able to maintain their visual acuity over the course of their treatment on the O\&P regimen. This is the first time that this has been demonstrated in established nAMD that has previously been treated with the PRN regime for many years: Many of our patients exhibited features of advanced age related macular degeneration. For example all had sub retinal fibrosis visible on the OCT scan at enrolment and $81 \%$ had a best corrected visual acuity below the driving standard.

It is hypothesised that multiple recurrent episodes of exudation, as occur with long term treatment under the PRN regimen, can exacerbate subretinal fibrosis [12]. A theoretical clinical advantage of the O\&P regimen over PRN when used for many years is a decrease in the rate of subretinal fibrosis as patients are treated pre-emptively with an interval short enough to prevent exudation rather than only after exudation has occurred. Whilst all of our patients already had at least some degree of subretinal fibrosis, we did demonstrate a statistically significant decrease in the percentage of patients with macular oedema.

The key strength of the O\&P regimen compared to PRN is in the number of clinical reviews required. Our findings within the PRN group corroborate previous work by demonstrating that the required treatment interval for patients with nAMD is relatively stable for each individual, whilst being highly variable between individuals [2]. This enables injections to be planned in groups based on an observation of the required treatment interval. In our study the benefit for patients was a reduction in the total number of visits to the eye clinic from 9.0 to 5.6 per year and in addition the patient received an injection at every visit, therefore there were no visits that were superfluous to the treatment requirements of the patient. The economic advantage comes chiefly from the reduced number of clinical reviews (requiring an appointment with an ophthalmologist). A reduction from 9.0 per year under PRN to 1.80 per year under O\&P was found, which equates to an economic saving of $£ 1,228$ per patient per year, or approximately $20 \%$ of the total cost of treatment.

Treatment of neovascular age related macular degeneration now accounts for approximately $0.1 \%$ to $0.2 \%$ of the entire NHS budget [13]. As the population ages the number of patients requiring treatment will continue to rise steeply. It is therefore imperative to find innovative strategies to reduce the cost burden whilst maintaining the efficacy of the treatment. In order to facilitate a rapid change in practice to more cost efficient regiments, evidence of non-inferiority is not only required for treatment naive patients, but crucially for the vast majority of patients who are already on treatment.

A principal strength of this study is that it is the first head to head comparison of O\&P versus an established treatment regimen. It is also a real world study and managed to recruit a very elderly population as compared to most clinical trials that tend to recruit a younger age group. This makes it more representative of patients seen in the clinic. In addition, the evidence for the effectiveness of observe and plan is extended to patients with longstanding, often advanced, nAMD who have previously been under the PRN regimen. Most patients were pseudophakic, removing media opacity as a confounding factor in the analysis.

The weakness of the study is in its small sample size. Despite this, statistically significant non-inferiority of O\&P as compared to PRN was still demonstrated. A larger study would be required to further investigate the safety profile of O\&P compared to PRN and to look for potential superiority of O\&P over PRN. In addition we analysed only patients undergoing treatment with aflibercept, rather than ranibizumab or avastin. Finally we did not compare O\&P to T\&E in this study.
In conclusion, the O\&P regimen for treatment of nAMD was clinically non-inferior to PRN. The O\&P regimen was superior to PRN in terms of reducing the health economic burden and treatment burden of nAMD.

\section{Conflict of interest}

The authors do not have any conflicts of interest to disclose in relation to the subject matter of this paper.

\section{Acknowledgements}

Mr Bobby Paul, Barking Havering and Redbridge University Hospitals Trust

\section{Trust}

Mr Khan, Barking Havering and Redbridge University Hospitals

\section{References}

1. Mantel I, Niderprim SA, Gianniou C, Deli A, Ambresin A (2014) Reducing the clinical burden of ranibizumab treatment for neovascular age-related macular degeneration using an individually planned regimen. Br J Ophthalmol 98: 1192-1196. [Crossref]

2. Mantel I, Deli A, Iglesias K, Ambresin A (2013) Prospective study evaluating the predictability of need for retreatment with intravitreal ranibizumab for age-related macular degeneration. Graefes Arch Clin Exp Ophthalmol 251: 697-704. [Crossref]

3. Gianniou C, Dirani A, Ferrini W, Marchionno L, Decugis D, et al. (2015) Twoyear outcome of an observe-and-plan regimen for neovascular age-related macular degeneration: how to alleviate the clinical burden with maintained functional results. Eye 29: 342-349. [Crossref]

4. Parvin P, Zola M, Dirani A, Ambresin A, Mantel I (2017) Two-year outcome of an observe-and-plan regimen for neovascular age-related macular degeneration treated with Aflibercept. Graefes Arch Clin Exp Ophthalmol 255: 2127-2134. [Crossref]

5. Owen CG, Jarrar Z, Wormald R, Cook DG, Fletcher AE, et al. (2012) The estimated prevalence and incidence of late stage age related macular degeneration in the UK. $B r$ J Ophthalmol 96: 752-756. [Crossref]

6. Amoaku W, Blakeney S, Freeman M, Gale R, Johnston R, et al. (2012) Action on AMD. Optimising patient management: act now to ensure current and continual delivery of best possible patient care. Eye 26: S2. [Crossref]

7. Solomon SD, Lindsley K, Vedula SS, Krzystolik MG, Hawkins BS (2019) Antivascular endothelial growth factor for neovascular age-related macular degeneration. Cochrane Database Syst Rev 8: CD005139.. [Crossref]

8. Bogunović H, Waldstein SM, Schlegl T, Langs G, Sadeghipour A, et al. (2017) Prediction of anti-VEGF treatment requirements in neovascular AMD using a machine learning approach. Invest Ophthalmol Vis Sci 58: 3240-3248. [Crossref]

9. Fung AE, Lalwani GA, Rosenfeld PJ, Dubovy SR, Michels S, et al. (2007) An optica coherence tomography-guided, variable dosing regimen with intravitreal ranibizumab (Lucentis) for neovascular age-related macular degeneration. Am J Ophthalmol 143: 566-583

10. Gillies MC, Hunyor AP, Arnold JJ, Guymer RH, Wolf S, et al. (2019) Effect of Ranibizumab and Aflibercept on Best-Corrected Visual Acuity in Treat-and-Extend fo Neovascular Age-Related Macular Degeneration: A Randomized Clinical Trial. JAMA Ophthalmol 137: 372-379. [Crossref]

11. Kaiser PK, Singer M, Tolentino M, Vitti R, Erickson K, et al. (2017) Long-term safety and visual outcome of intravitreal aflibercept in neovascular age-related macula degeneration: VIEW 1 extension study. Ophthalmol Retina 1: 304-313. [Crossref]

12. Daniel E, Toth CA, Grunwald JE, Jaffe GJ, Martin DF, et al. (2013) Risk of scar in the comparison of age-related macular degeneration treatments trials. Ophthalmology 121: 656-666. [Crossref]

13. Dakin HA, Wordsworth S, Rogers CA, Abangma G, Raftery J, et al. (2014) Costeffectiveness of ranibizumab and bevacizumab for age-related macular degeneration: 2-year findings from the IVAN randomised trial. BMJ Open 4: e005094.

Copyright: (C)2020 Wawrzynski J. This is an open-access article distributed under the terms of the Creative Commons Attribution License, which permits unrestricted use, distribution, and reproduction in any medium, provided the original author and source are credited. 